\title{
Exciton-Exciton Annihilation in Two-Dimensional Halide Perovskites at Room Temperature
}

\author{
Géraud Delport, ${ }^{\dagger}$ Gabriel Chehade, ${ }^{\dagger}$ Ferdinand Lédée, $^{\dagger}$ Hiba Diab, ${ }^{\dagger}$ Cosme \\ Milesi-Brault, ${ }^{\dagger}$ Gaëlle Trippé-Allard, ${ }^{\dagger}$ Jacky Even, ${ }^{\dagger}$ Jean-Sébastien Lauret, ${ }^{\dagger}$ \\ Emmanuelle Deleporte, ${ }^{\dagger}$ and Damien Garrot*, \\ $\dagger$ †aboratoire Aimé Cotton, CNRS, Université Paris-Sud, ENS Paris-Saclay, Université \\ Paris-Saclay, 91405 Orsay Cedex, France \\ $\ddagger$ Univ Rennes, INSA Rennes, CNRS, Institut FOTON - UMR 6082, Rennes F-35000, \\ France \\ \Groupe d'Etude de la Matière Condensée, CNRS, Université de Versailles \\ Saint-Quentin-en-Yvelines, Université Paris-Saclay, 45 Avenue des Etats-Unis, 78035, \\ Versailles, France \\ E-mail: damien.garrot@uvsq.fr
}

\section{Abstract}

Recently, Ruddlesden-Popper 2D perovskites (RPPs) solar cells and Light-Emitting Diodes (LED) have shown promising efficiencies and improved stability in comparison to $3 \mathrm{D}$ halide perovskites. Here, the exciton recombination dynamics is investigated at room temperature in pure phase RPPs crystals $\left(\mathrm{C}_{6} \mathrm{H}_{5} \mathrm{C}_{2} \mathrm{H}_{4} \mathrm{NH}_{3}\right)_{2}\left(\mathrm{CH}_{3} \mathrm{NH}_{3}\right)_{\mathrm{n}-1} \mathrm{~Pb}_{\mathrm{n}} \mathrm{I}_{3 \mathrm{n}+1} \quad(\mathrm{n}=1$, 2, 3 and 4) by time-resolved photoluminescence in a large range of power excitations. As the number of perovskite layers increases, we detect the presence of an increasing fraction of out-of-equilibrium free carriers just after photoexcitation, on a picosecond timescale, while the dynamics is characterized by the recombination of excitons with long lifetime spanning on several tens of nanoseconds. At low excitation power, the PL decays are non-exponential due to defect-assisted recombination. At high fluence, defects are filled and many body interactions become important. Similarly to other 2D systems, Exciton-Exciton Annihilation (EEA) is then the dominant recombination path in a high density regime below the Mott transition.

\section{Graphical TOC Entry}

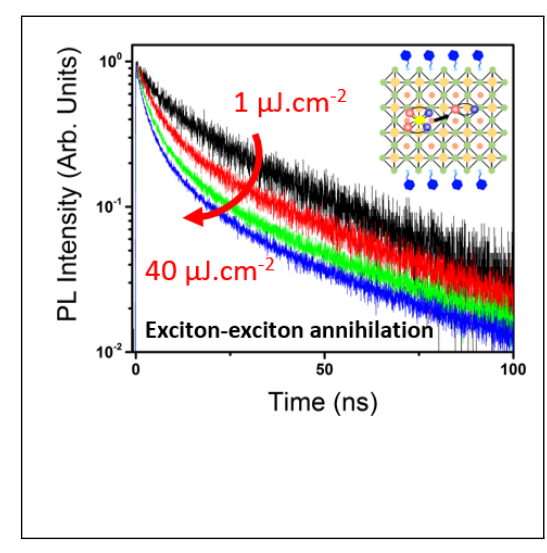

\section{Keywords}

Halide Perovskite, Ruddlesden-Popper Phase, Exciton Dynamics, Defect, Exciton-Exciton Annihilation 
Two-dimensional Ruddlesden-Popper perovskites present the general formula $\left(\mathrm{RNH}_{3}\right)_{2}(\mathrm{MA})_{n-1} \mathrm{~A}_{n} \mathrm{X}_{3 n+1}$ where $\mathrm{MA}=\mathrm{CH}_{3} \mathrm{NH}_{3}$, $\mathrm{R}$ is an alkyl or aromatic group, $\mathrm{M}$ is metal and $\mathrm{X}$ is halide. They formed self-assembled twodimensional structures with $\mathrm{n} \mathrm{AX}_{6}$ octahedra layers separated by organic molecules. Quantum and dielectric confinement leads to the formation of strongly bound excitons. ${ }^{1,2}$ The electronic properties can be tuned by increasing the number of inorganic layers from $2 \mathrm{D}$ to $3 \mathrm{D}(\mathrm{n}=\infty) \cdot{ }^{3-5}$

Recently, RPPs have attracted great attention for solar cells applications since a breakthrough in 2016 in the growth procedure of pure RPPs compounds ${ }^{6}$ and their use in $2 \mathrm{D} / 3 \mathrm{D}$ mixed solar cell structures. ${ }^{7}$ In both cases, a greater long-term stability than their 3D counterpart was demonstrated. Additionally, RPPs are also particularly promising for their emission properties. Light-emitting diodes (LED) have been realized with mixed phase ${ }^{8,9}$ and pure phase RPPs. ${ }^{10}$ Importantly, the latters present a greater operational stability. Amplified Spontaneous Emission (ASE) with low threshold has been reported in mixed phase RPPs, due to the efficient energy transfer from high band-gap to low band-gap phases where carriers are in consequence concentrated. ${ }^{11,12}$ Interestingly, low threshold lasing has been recently observed in pure phase RPP single crystals based on the butylammonium (BA) cation. ${ }^{13}$

Most of the studies on the carrier dynamics focused on mixed phase thin films for which carriers properties are very dependent of the specific distribution of phases due to energy/charge transfer. ${ }^{14-21}$ To the best of our knowledge, only a few reports have investigated the recombination dynamics in pure phase $\operatorname{RPPs}^{22-24}$ and only one on the high excitation regime using pump-probe spectroscopy, but focusing on the sub-ns time range. ${ }^{25}$

To optimize the performance of devices, a better understanding of the recombination mechanisms and sources of non-radiative lossses is necessary. Many-body effects are enhanced in low dimensional materials. In particular, Exciton-Exciton Annihilation is a two parti- cles interaction in which an exciton recombines nonradiatively to the ground state and transfer its energy to a second exciton. The process is analog to the three-body Auger interaction for free carriers. It has been studied extensively in carbon nanotubes, ${ }^{26,27}$ quantum dots, ${ }^{28,29}$ nanowires $^{30,31}$ and $2 \mathrm{D}$ materials. ${ }^{32-35}$ EEA and Auger recombination are limiting factors for carrier injection in light-emitting devices. $^{36-38}$ Interestingly, efficient EEA implies that the inverse process, multiple exciton generation, could also be effective and lead to an increase of the solar cells energy conversion efficiency beyond the Shockley-Queisser limit. ${ }^{39,40}$

Here, we study the exciton recombination in pure phase $\mathrm{PEA}$-based $(\mathrm{PEA}=$ phenylethylammonium $\left.\mathrm{C}_{6} \mathrm{H}_{5} \mathrm{C}_{2} \mathrm{H}_{4} \mathrm{NH}_{3}^{+}\right)$RPPs crystals $(\mathrm{PEA})_{2}(\mathrm{MA})_{n-1} \mathrm{~Pb}_{n} \mathrm{I}_{3 n+1}$ $(\mathrm{n}=1,2,3$ and 4$)$ using Time-Resolved Photoluminescence (TRPL) measured in a large range of excitation powers. The excitation in the continuum leads to the rapid formation of excitons. However, for $n>1$, a remaining fraction of free carriers in a non-equilibrium state is detected on a picosecond timescale just after excitation. Then, the emission mechanism is dominated by excitons. At low fluence, the PL decay is nonexponential and dependent of the excitation density due to the presence of non-radiative recombination centers (NRCs). In particular, a sub-nanosecond component associated to defect-assisted recombination is identified for $n<4$. The density of defects increases with lower number of layers. At higher excitation power, many body effects can not be neglected and the recombination dynamics is successfully described by an EEA model.

The schematic structures of $(\mathrm{PEA})_{2}(\mathrm{MA})_{n-1} \mathrm{~Pb}_{n} \mathrm{I}_{3 n+1}$ $(\mathrm{n}=1,2,3$ and 4$)$ crystals are shown in Figure 1a. Figure 1b shows the PL spectra of the synthetized crystals (Figure 1c) at room temperature (RT). The emission of each crystals is characterized by a single peak, at the expected energy position for $(\mathrm{PEA})_{2}(\mathrm{MA})_{n-1} \mathrm{~Pb}_{n-1} \mathrm{I}_{3 n+1}$ single crystals. ${ }^{24,41}$ We note that in the presence of a mixture of different phases the emission of RPPs is characterized by multiple peaks, and the predominance of the lowest energy peak due to the fast transfer of energy from the small$\mathrm{n}$ to the lowest bandgap phase (large $\mathrm{n}$ ). ${ }^{8,9}$ 

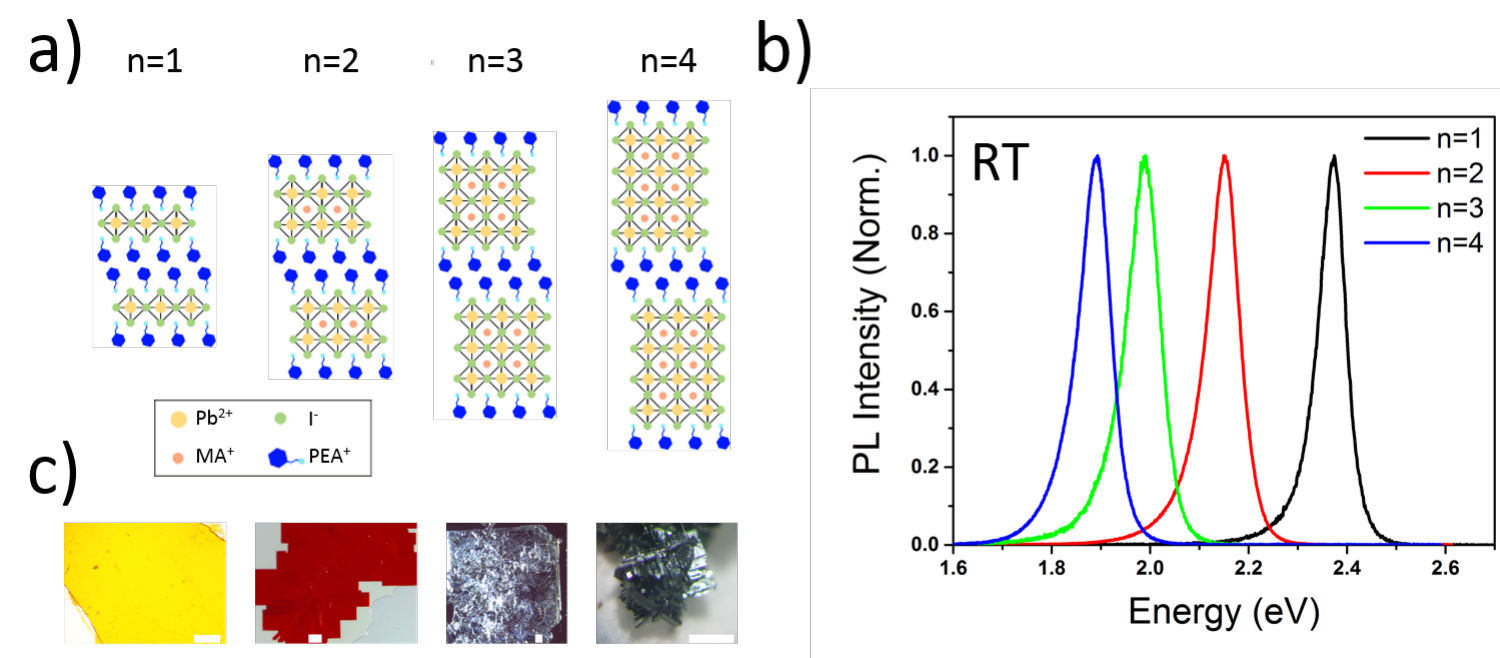

Figure 1: a) Schematic representation of $(\mathrm{PEA})_{2}(\mathrm{MA})_{n-1} \mathrm{~Pb}_{n} \mathrm{I}_{3 n+1}(\mathrm{n}=1,2,3$ and 4$)$ structures b) Room temperature photoluminescence spectra of RPP crystals from $n=1$ to 4 c) Optical images of crystals of the same RPP materials, scale bars represent $100 \mu \mathrm{m}$

Hence, the PL spectra reported in this study are clearly indicative of pure phase crystals of $(\mathrm{PEA})_{2}(\mathrm{MA})_{n-1} \mathrm{~Pb}_{n} \mathrm{I}_{3 n+1}(\mathrm{n}=1,2,3$ and 4$)$.

We observe a progressive blueshift of the emission as $\mathrm{n}$ goes from 4 to 1 , as expected from the increase of the band gap due to quantum confinement. ${ }^{42}$ In RPPs, the exciton binding energy $\left(\mathrm{E}_{b}\right)$ depends on the $\mathrm{n}$ value and increases with the dielectric and quantum confinement. $E_{b}$ has been estimated experimentally to $220 \mathrm{meV}$ for $\mathrm{n}=1$ and $170 \mathrm{meV}$ for $\mathrm{n}=2$ PEA based RPPs. ${ }^{4,43}$ For $\mathrm{n}=3$ and $\mathrm{n}=4$, we estimate $\mathrm{E}_{b}$ to approximately $125 \mathrm{meV}$ and $100 \mathrm{meV}$ using the scaling law recently proposed by Blancon et al. based on their study of $(\mathrm{BA})_{2}(\mathrm{MA})_{n-1} \mathrm{~Pb}_{n} \mathrm{I}_{3 n+1}$ (See Figure S2 and SI for details). ${ }^{2}$

To assess the recombination mechanisms in RPPs, we measured the TRPL dynamics after a non-resonant excitation at $3.1 \mathrm{eV}$, using a large range of excitation powers. We observe a nonexponential dynamics for the four compounds, with the slowest component of the order of several tens of nanoseconds (Figure S3). In comparison, mono-exponential decays with a characteristic PL lifetime of only 1-2 ns have been reported for exfoliated crystals with $n=1,2,3 .{ }^{23}$ The PL dynamics present a complex evolution as function of $n$ value and excitation power.

In order to analyze these PL decays, first we report $\mathrm{PL}_{0}$, the $\mathrm{PL}$ intensity at $\mathrm{t}=0 \mathrm{~ns}$, at the instant of the pulse excitation, as a function of excitation power density for $n=1,2,3$ and 4 (Figure 2). The scaling of $\mathrm{PL}_{0}$ with fluence is classically used to analyze the nature of the radiative recombination processes. ${ }^{45}$ For the purely $2 \mathrm{D}$ compound $(\mathrm{n}=1), \mathrm{PL}_{0}$ is linear with the excitation density as it is expected for exciton recombination (monomolecular process). Upon excitation in the continuum, an unbound electron-hole pair is initially created before rapidly relaxing to lower energies and forming an exciton. For $n=1$, an ultrafast relaxation of approximately 150 fs has been measured. ${ }^{46}$

For $\mathrm{n}>1$, we observe a clear non-linearity, increasing with $\mathrm{n}$ from 1.2 to 1.7 for $\mathrm{n}=4$. For the $3 \mathrm{D}$ perovskites $\mathrm{CH}_{3} \mathrm{NH}_{3} \mathrm{PbI}_{3}$ (MAPI) a quadratic dependence $(\lambda=2)$ with excitation fluence has been observed, showing that the emission arises from the recombination of free carriers, a bimolecular process. ${ }^{47,48}$ Hence, we interpret the nonlinearity of $\mathrm{PL}_{0}$ for $n>1$ as due to the presence of a remaining fraction of free carriers, having not yet formed excitons, within our time resolution of approximately 100 ps (Figure S1). The increasing non-linearity is consistent with a decrease of the exciton binding energy with the number of inorganic layers. Due to the large excitonic binding energy of $2 \mathrm{D}$ perovskites, the photoexcited system will 


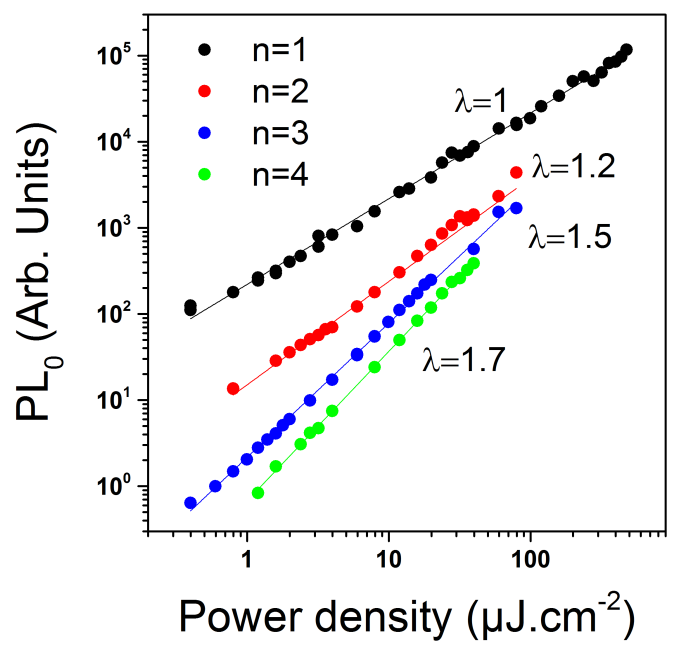

Figure 2: Room temperature photoluminescence emission intensity at $\mathrm{t}=0 \mathrm{~ns}\left(\mathrm{PL}_{0}\right)$ as a function of fluence for $n=1,2,3$ and 4 . The power law dependence is shown by continuous line. The relative values of intensities for $n=3$ have been vertically shifted for clarity.

evolve subsequently toward an excitonic equilibrium state before recombination, as it has been demonstrated in BA based RPPs. ${ }^{22,25,49}$

We turn now to the PL dynamics. The recombination dynamics for $n=1,2$ and 3 show a subnanosecond initial decay and a slower dynamics which spans over several tens of nanoseconds (Figure $3 \mathrm{a}, \mathrm{b}, \mathrm{c}$ and $\mathrm{S} 2$ ). For $\mathrm{n}=4$ crystals, only the slow component is observed. The nonexponential nature of the decay is indicative of the existence of different populations of excitons, spatially separated. ${ }^{50}$ We suppose that the population of excitons in layers close to the surface experiences a higher density of defects than excitons in deeper layers and are associated with the faster recombination rate. Indeed, surface relaxation effects leading to defects formation have been recently pointing out in $2 \mathrm{D}$ perovskites. ${ }^{51}$

As the fluence increases, the amplitude of the fast component tends to decrease (Figure 3a, b, c) and the lifetime increases (Figure S5), which is consistent with the progressive saturation of non-radiative recombination centers. The latter should cause an increase of the photoluminescence Quantum Yield (QY). The TimeIntegrated Photoluminescence (TIPL) and rel-
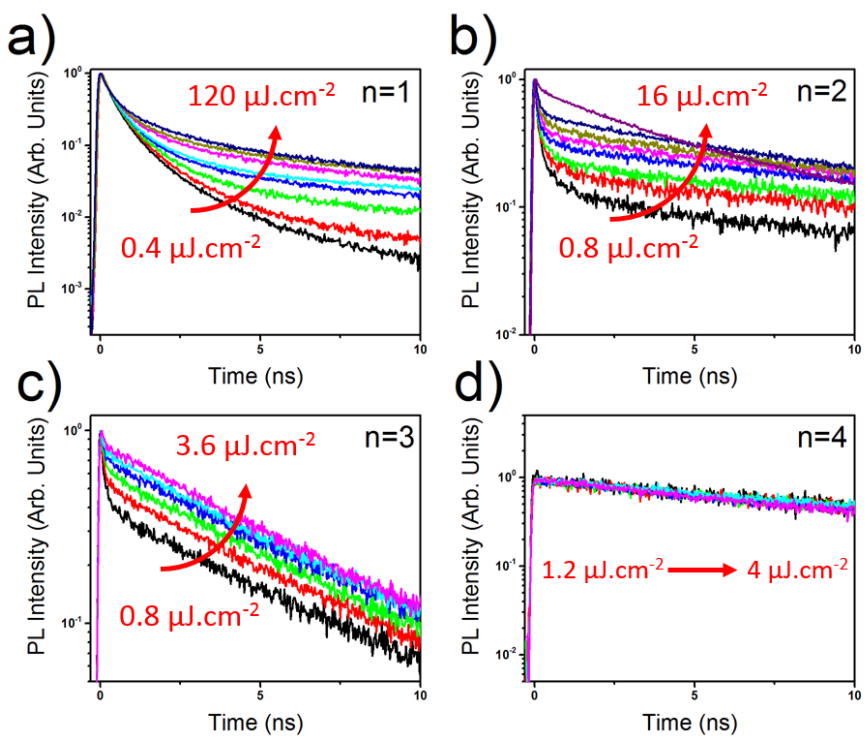

Figure 3: Evolution of PL decays as function of excitation power on a $10 \mathrm{~ns}$ timescale a) for $n=1$ b) $n=2$ and c) $n=3$ d) $n=4$ (curves obtained at longer time scales (100 ns) are shown on figure S3)

ative QY (defined as TIPL/P where $\mathrm{P}$ is the power density) are displayed in Figure 4 . The injected carrier density is reported on the top $\mathrm{x}$-axis. The QY has been normalized at its maximum value. A surlinear variation of the TIPL and an initial increase of the QY with excitation power is indeed observed which confirms the assignation of the sub-nanosecond decay to NRCs and the longer lifetime to the effective exciton lifetime. This is consistent with our observation of a shorter lifetime on polycrystalline $\mathrm{n}=1$ thin films than crystals (Figure S4). ${ }^{46} \mathrm{Ad}-$ ditionally, a recent study on $n=1$ PEA based crystals indicates that light degradation causes an increase of the fast component, which confirms its extrinsic nature. ${ }^{52}$

After the fast initial decay, the PL dynamics is exponential as expected for excitonic recombination (Figure S6). Remarkably, a lower excitation power is necessary to suppress the fast decay as $n$ increases. For $n=4$, there is no sub-nanosecond component and the PL decay is nearly exponential and independent of the injection density at low fluence (Figure 3d) which is consistent with the recombination of excitons. Hence, the density of NRCs specifically associated with the sub-nanosecond com- 

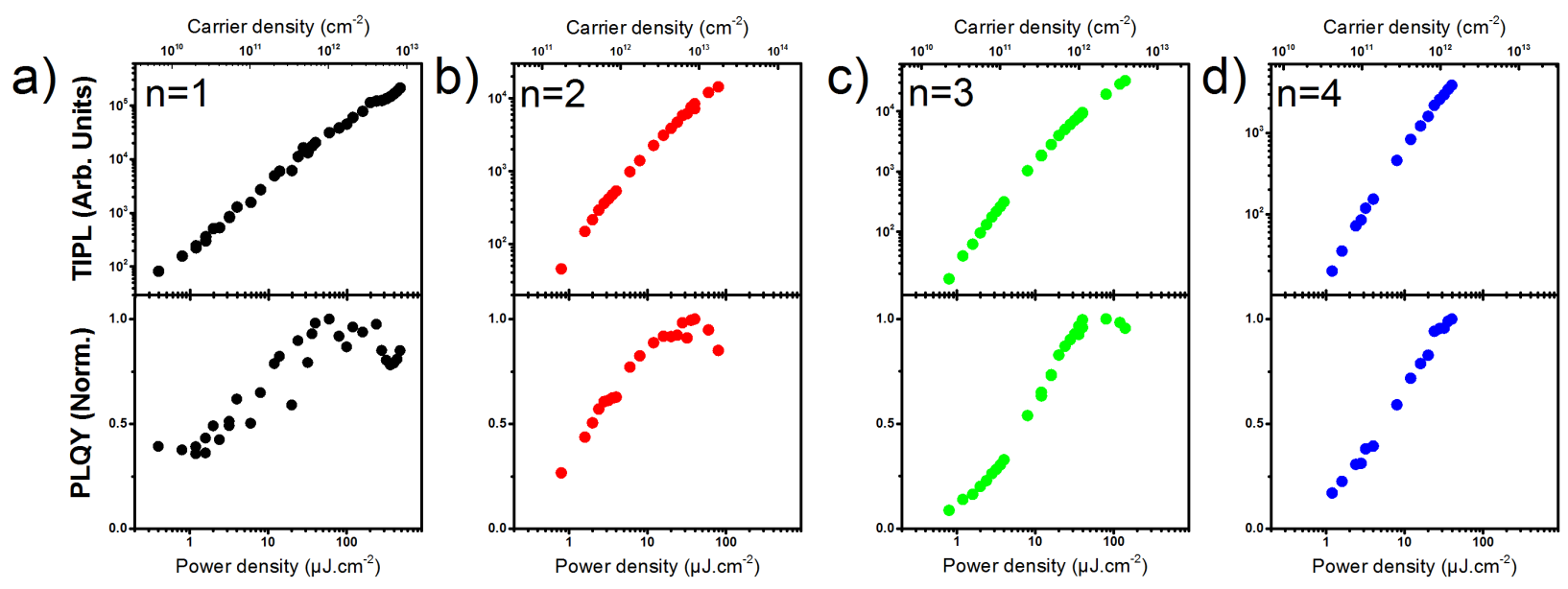

Figure 4: a) Time-integrated PL and normalized quantum yield as function of fluence for a) $n=1$ b) 2 c) 3 and d) 4 . Top x-axis, estimated carrier density

ponent, seems to decrease with increasing perovskites layers. The observation is in good agreement with the study of X.-Y. Zhu and coworkers on BA based RPPs thin films. ${ }^{53} \mathrm{We}$ note however that the increase of the QY with power excitation for $n=4$ is indicative of the presence and saturation of traps in this compound.

The evolution of the PL decays is continuous from $n=2$ to $n=4$, however it changes dramatically going from $n=1$ to $n=2$ (Figure $3 b$, $c$ and $\mathrm{S} 2$ ). In particular, for $\mathrm{n}=2$ and $\mathrm{n}=3$, the fast initial decay presents a characteristic time of approximately $120 \mathrm{ps}$ in comparison to $400 \mathrm{ps}$ for $n=1$. It is important to note that not only the width of the inorganic layer (the well) is increased but $\mathrm{CH}_{3} \mathrm{NH}_{3}{ }^{+}$organic cations are also added to the structure. The latters may interact with the charges through electric field and impact the recombination dynamics. ${ }^{54}$

Increasing the excitation power further, we observe a new regime characterized by an increasing recombination rate for all compounds. For $\mathrm{n}=1$, at excitation power superior to 100 $\mu \mathrm{J} . \mathrm{cm}^{-2}$ ( carrier density $>10^{12} \mathrm{~cm}^{-2}$ ), both the lifetime and QY decrease. We note that at the carrier density used here, the 2D massaction law, known as Saha equation, which describes the equilibrium between free and correlated electron-hole pairs, indicates the formation of a population of excitons (Figure S7). At a density of $10^{12} \mathrm{~cm}^{-2}$, the average excitonexciton separation, $\frac{2}{\sqrt{n \pi}}$ is inferior to $10 \mathrm{~nm}$. A rough upper estimate of the Mott transition density is given by $n_{m} \approx a_{B}^{-2}$ where $a_{B}$ is the $2 \mathrm{D}$ Bohr radius of the exciton. ${ }^{55}$ Beyond the Mott transition, the photoexcited species cannot be described as excitons, but form an electron-hole plasma. In a previous study, we estimate $a_{B}$ to $1.5 \mathrm{~nm}^{56}$ which gives $n_{m} \approx 3.5 \times 10^{13} \mathrm{~cm}^{-2}$. Hence, in the highest excitation range, we are in an intermediate density regime where excitons are still good quasi-particles, but where many-body effects may be important. Among these effects, the formation of biexcitons has been reported in $n=1 \operatorname{RPPs}^{57-59}$ with binding energy varying between 40 and $60 \mathrm{meV}$ and may cause a shorter lifetime of excitonic emission. We have observed no additional line which would correspond to biexciton formation in the PL spectra or in transient absorption, even at a high density of $10^{13} \mathrm{~cm}^{-2}$. ${ }^{46}$ This might be consistent with the fact that biexciton lasing was only observed at low temperature. ${ }^{60}$

Hence, we assigned the faster recombination rate and the QY decrease to Exciton-Exciton Annihilation (EEA). At high fluence, the kinetics equation can be written as :

$$
\frac{d n_{x}}{d t}=-\frac{n_{x}}{\tau}-\gamma n_{x}^{2}
$$

where $n_{x}$ is the exciton density, $\tau$ is the exci- 
ton recombination lifetime in absence of EEA, defined as $\frac{1}{\tau}=\frac{1}{\tau_{n r}}+\frac{1}{\tau_{r}}$ with $\tau_{n r}$ and $\tau_{r}$ the nonradiative and radiative lifetime respectively. $\gamma$ is the EEA rate constant. We assume here that the annihilation rate is time-independent. The resolution of equation 1 yields to :

$$
n_{x}(t)=\frac{n_{x}(0) \exp (-t / \tau)}{1+\gamma \tau n_{x}(0)(1-\exp (-t / \tau))}
$$

Due to the effect of traps saturation, $\tau$ could not be fixed from low fluence measurements and is introduced as a fitting parameter. The recombination model applied on the PL decays at the highest fluence leads to an excellent result (Figure 5a), with coefficient of determination $R^{2}=0.999$ on more than three orders of magnitude on the PL intensity. We obtain an exciton lifetime of $\tau=(28 \pm 7)$ ns and an annihilation rate of $3.35 \times 10^{-4} \mathrm{~cm}^{2} \mathrm{~s}^{-1}$.
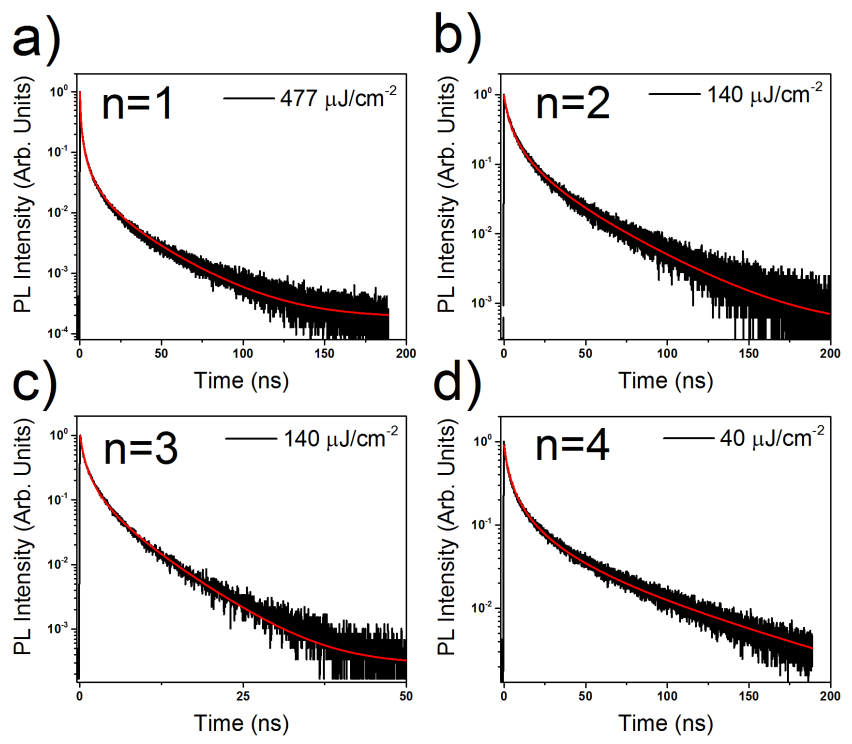

Figure 5: a) PL decays at high excitation power and recombination model according to equation 1 (red lines) for a) $n=1$ b) $n=2$ c) $n=3$ d) and $\mathrm{n}=4$ RPPs crystals

The PL dynamics at high excitation power has also been fitted with equation 1 for $n=2,3$ and 4, leading again to an excellent agreement with the recombination model (Figure 5b, c and d). Fit parameters for the different crystals are resumed in Table 1 . For $n>1$, the PL lifetime decreases from a lower injection density of approximately $10^{11} \mathrm{~cm}^{-2}$ (Figure $\mathrm{S} 5$ ) in compari- son to $n=1$. The $Q Y$ reaches a plateau and for $\mathrm{n}=2$ and 3 starts to decrease. We interpret the general evolution of the QY as the competition between the effect of NRCs saturation, reached at lower carrier injection as $\mathrm{n}$ increases, and the occurence of EEA.

A description of the recombination dynamics in term of excitonic recombination gives a very good agreement with the measurements. We note however that for $n=4$ crystals, the mass action law indicates, at the lower power excitation used in this study, a non-negligible fraction of free carriers of approximately $10 \%$ (Figure S7). At even lower power excitations, compatible with sun irradiation, free carriers may play a significant role in the photophysics of RPPs, especially for $n=4$ crystals. ${ }^{17,20,24}$ Hence, our results are not incompatible with the performance of $n \geq 4$ RPPs in photovoltaic applications, which implies an efficient charge separation. ${ }^{6,61}$

Table 1: list of fit parameters from equation 1 for $\mathrm{n}=1,2,3$ and 4 (see Figure 5)

\begin{tabular}{lll}
\hline & $\tau(\mathrm{ns})$ & $\gamma\left(\mathrm{cm}^{2} \mathrm{~s}^{-1}\right)$ \\
\hline $\mathrm{n}=1$ & $28 \pm 7$ & $(3.35 \pm 0.15) \times 10^{-4}$ \\
$\mathrm{n}=2$ & $35 \pm 4$ & $(1.10 \pm 0.35) \times 10^{-4}$ \\
$\mathrm{n}=3$ & $6.7 \pm 0.8$ & $(4.90 \pm 0.12) \times 10^{-4}$ \\
$\mathrm{n}=4$ & $80 \pm 10$ & $(2.50 \pm 0.50) \times 10^{-4}$
\end{tabular}

The exciton lifetime increases with the $\mathrm{n}$ value, with the exception of $n=3$ crystals. An increase of the radiative lifetime is expected due to the fact that, in a quantum well, the excitonic lifetime is inversely proportional to $\mathrm{E}_{b} .{ }^{62}$ However, as discussed previously, we have identified in this study different defect-assisted non-radiative recombination channels. Regarding $n=3$ RPPs, pure phase crystals could not be obtained with the anti-solvant method (see experimental section in SI), and instead have been synthesized with the slow cooling method. The latter seems to induce a higher density of defects and in consequence a shorter lifetime. For the other crystals, we observe long exciton lifetimes spanning on several tens of nanoseconds. Long exciton lifetimes at room temperature have also been observed in transition metal dichalcogenide (TMD) monolayers despite large excitonc binding energies of several hundred of 
meV. ${ }^{63}$ Effective lifetime as long as $10 \mathrm{~ns}$ has been measured for example in $\mathrm{MoS}_{2}$ monolayers at room temperature. ${ }^{64} \mathrm{~A}$ radiative lifetime of $100 \mathrm{~ns}$ has been estimated in $\mathrm{WSe}_{2} \cdot{ }^{65}$

We have identified two excitation-dependent, non-radiative recombination channels. Defectassisted recombination is efficient at low excitation power, while EEA becomes dominant at high fluence. Regarding EEA, the annihilation rates do not present strong variation as the number of layers increases. For $n>1$, the onset of EEA is detected at a lower density $\left(\approx 10^{11} \mathrm{~cm}^{-2}\right)$ than for $\mathrm{n}=1$, because of a lower density of defects. For $n=1$, defectassisted recombination is still present at high fluence. Hence, EEA is measured at excitation power $\mathrm{P}>400 \mu \mathrm{J} . \mathrm{cm}^{-2}$ for which the annihilation effective lifetime $\left(\gamma n_{x}\right)^{-1}$ becomes inferior to $400 \mathrm{ps}$ and compete efficiently with defectassisted recombination lifetime. In the absence of defects, the onset of EEA would likely be detected at lower carrier density for $n=1$. Similar competition between EEA and defectassisted recombination has been observed in other 2D materials. ${ }^{35,66}$ In TMD monolayers, annihilation rates has been reported to be $0.04 \mathrm{~cm}^{2} \mathrm{~s}^{-1}$ in $\mathrm{MoS}_{2}, 0.33 \mathrm{~cm}^{2} \mathrm{~s}^{-1}$ in $\mathrm{MoSe}_{2}$ and $0.41 \mathrm{~cm}^{2} \mathrm{~s}^{-1}$ in $\mathrm{WS}_{2} \cdot{ }^{32-34}$ A lower annihilation rate of $5.10^{-3} \mathrm{~cm}^{2} \mathrm{~s}^{-1}$ has been reported in black phosphorus. ${ }^{35}$ The annihilation rate depends on the diffusion constant and on the typical distance between excitons at which EEA occurs. The high annihilation rates reported for TMDs are due to an important exciton mobility, with an exciton diffusion constant of approximately $10 \mathrm{~cm}^{2} \mathrm{~s}^{-1} \cdot{ }^{67}$ In pure phase RPPs, further studies are needed as the exciton diffusion constant is still unknown.

Importantly, Amplified Spontaneous Emission (ASE) or lasing is not observed in this study, whereas lasing has been reported in a recent study by Raghavan et al. in pure phase BA based RP crystals, with a threshold of approximately $3 \mu \mathrm{J} . \mathrm{cm}^{-2}$ under similar conditions to the one used in the present study. ${ }^{13}$ Annihilation rates have not yet been reported in $\mathrm{BA}-$ RPPs. We note that, according to our observations, a power excitation of $3 \mu \mathrm{J} . \mathrm{cm}^{-2}$ corresponds approximately to the onset of EEA in
PEA based RPPs. The larger excitonic binding energy of BA based RPPs ${ }^{2}$ may promote a faster excitonic recombination competing efficiently with non-radiative recombination channels. It highlights the role of the organic cation and the importance of dielectric confinement. Additionally, the bulkier PEA cation may also induce more disorder and strain inside the material leading to superior non-radiative losses.

The nature of defects in RPPs is not well known. Wu et al. have suggested an intrinsic origin of trap states caused by electron-phonon coupling at the surfaces/interfaces, with the formation of self-trapped excitons/carriers. ${ }^{53}$ However, here, we highlight a saturation effect more compatible with the formation of chemical defects. Two recent theoretical studies have concluded that in $2 \mathrm{D}$ halide perovskites $(\mathrm{n}=1)$ defects induce only shallow states and not deep gap states. ${ }^{68,69}$ Despite the predicted defect tolerance, our observations show the presence of a large density of defects, especially for perovskite monolayers, filled only at high excitation power. The results suggest that the passivation of traps are necessary to optimize the optoelectronics properties of RPPs.

In conclusion, we have studied the recombination dynamics of low dimensional

$(\mathrm{PEA})_{2}\left(\mathrm{CH}_{3} \mathrm{NH}_{3}\right)_{\mathrm{n}-1} \mathrm{~Pb}_{\mathrm{n}} \mathrm{I}_{3 \mathrm{n}+1} \quad(\mathrm{n}=1,2,3$ and 4$)$ crystals at room temperature in a large range of excitation powers. The non-resonant photoexcitation creates unbound electron-hole pairs which relaxes rapidly to form excitons. For $\mathrm{n}>1$, we observe an increasing fraction of outof-equilibrium free carriers just after excitation, within our resolution of approximately 100 ps. The recombination mechanism is excitonic and the PL dynamics is highly dependent of the fluence for all the crystals due the effect of defects saturation and EEA. Non-radiative recombination centers causing a fast sub-nanosecond decay have been identified, with a decreasing density as $\mathrm{n}$ increases. We have observed long excitonic effective lifetime ranging from 30 to 80 ns. At high fluence, defects are being progressively filled and the dynamics is dominated by EEA. Annihilation rates are of the order of $10^{-4} \mathrm{~cm}^{2} \mathrm{~s}^{-1}$ and depend weakly of the number of perovskite layers. ASE or lasing are not 
observed which we attribute to the presence of defects and disorder in the material, leading to non-radiative losses. These results have major implications for the optimization of optolectronics devices and in particular light-emitting devices.

Acknowledgement This project has received funding from the European Union's Horizon 2020 programme, through a FET Open research and innovation action under the grant agreement No 687008. The information and views set out in this article are those of the author(s) and do not necessarily reflect the official opinion of the European Union. Neither the European Union institutions and bodies nor any person acting on their behalf may be held responsible for the use which may be made of the information contained herein. The work is supported by Agence Nationale pour la Recherche (EMIPERO project). ANR-10-04) J.S. Lauret is a junior member of the Institut Universitaire de France. J. Even is a senior member of the Institut Universitaire de France.

\section{Supporting Information Avail- able}

Experimental Methods, Carrier density calculation, Estimation of exciton binding energy, TRPL decays, Comparison between crystal and thin films PL decay, Average lifetime, Exponential fits, Mass action law calculations

\section{References}

(1) Ishihara, T.; Takahashi, J.; Goto, T. Exciton state in two-dimensional perovskite semiconductor $\left(\mathrm{C}_{10} \mathrm{H}_{21} \mathrm{NH}_{3}\right)_{2} \mathrm{PbI}_{4}$. Solid State Commun. 1989, 69, 933-936.

(2) Blancon, J.-C.; Stier, A. V.; Tsai, H.; Nie, W.; Stoumpos, C. C.; Traoré, B.; Pedesseau, L.; Kepenekian, M.; Katsutani, F.; Noe, G. T. et al. Scaling law for excitons in 2D perovskite quantum wells. Nat. Commun. 2018, 9.
(3) Calabrese, J.; Jones, N. L.; Harlow, R. L.; Herron, N.; Thorn, D. L.; Wang, Y. Preparation and Characterization of Layered Lead Halide Compounds. J. Am. Chem. Soc. 1991, 113, 2328-2330.

(4) Hong, X.; Ishihara, T.; Nurmikko, A. V. Dielectric Confinement Effect On Excitons In Pbi4-based Layered Semiconductors. Phys. Rev. B 1992, 45, 6961-6964.

(5) Papavassiliou, G.; Patsis, A.; Lagouvardos, D.; Koutselas, I. Spectroscopic Studies Of $\left(\mathrm{C}_{10} \mathrm{H}_{21} \mathrm{NH}_{3}\right)_{2} \mathrm{PbI}_{4}$, $\left(\mathrm{CH}_{3} \mathrm{NH}_{3}\right)\left(\mathrm{C}_{10} \mathrm{H}_{21} \mathrm{NH}_{3}\right)_{2} \mathrm{~Pb}_{2} \mathrm{I}_{7}$, $\left(\mathrm{CH}_{3} \mathrm{NH}_{3}\right) \mathrm{PbI}_{3}$, And Similar Compounds. Synth. Met. 1993, 57, 3889-3894.

(6) Tsai, H.; Nie, W.; Blancon, J.-C.; Stoumpos, C. C.; Asadpour, R.; Harutyunyan, B.; Neukirch, A. J.; Verduzco, R.; Crochet, J. J.; Tretiak, S. et al. High-efficiency two-dimensional Ruddlesden-Popper perovskite solar cells. Nature 2016, 536, 312-316.

(7) Quan, L. N.; Yuan, M.; Comin, R.; Voznyy, O.; Beauregard, E. M.; Hoogland, S.; Buin, A.; Kirmani, A. R.; Zhao, K.; Amassian, A. et al. LigandStabilized Reduced-Dimensionality Perovskites. J. Am. Chem. Soc. 2016, 138, 2649-2655.

(8) Yuan, M.; Quan, L. N.; Comin, R.; Walters, G.; Sabatini, R.; Voznyy, O.; Hoogland, S.; Zhao, Y.; Beauregard, E. M.; Kanjanaboos, P. et al. Perovskite energy funnels for efficient light-emitting diodes. Nat. Nanotechnol. 2016, 11, 872-877.

(9) Wang, N.; Cheng, L.; Ge, R.; Zhang, S.; Miao, Y.; Zou, W.; Yi, C.; Sun, Y.; Cao, Y.; Yang, R. et al. Perovskite light-emitting diodes based on solutionprocessed self-organized multiple quantum wells. Nat. Photonics 2016, 10, 699-704.

(10) Tsai, H.; Nie, W.; Blancon, J.-C.; Stoumpos, C. C.; Soe, C. M. M.; Yoo, J.; 
Crochet, J.; Tretiak, S.; Even, J.; Sadhanala, A. et al. Stable Light-Emitting Diodes Using Phase-Pure RuddlesdenPopper Layered Perovskites. Adv. Mater. 2018, 1704217.

(11) Li, M.; Gao, Q.; Liu, P.; Liao, Q.; Zhang, H.; Yao, J.; Hu, W.; Wu, Y.; Fu, H. Amplified Spontaneous Emission Based on 2D Ruddlesden-Popper Perovskites. Adv. Funct. Mater. 2018, 1707006.

(12) Leyden, M. R.; toshinori matsushima,; Qin, C.; Ruan, S.; Ye, H.; Adachi, C. Amplified Spontaneous Emission in Phenylethylammonium Methylammonium Lead Iodide Quasi-2D Perovskites. Phys. Chem. Chem. Phys. 2018, 20, 15030-15036.

(13) Raghavan, C. M.; Chen, T.-P.; Li, S.S.; Chen, W.-L.; Lo, C.-Y.; Liao, Y.M.; Haider, G.; Lin, C.-C.; Chen, C.-C.; Sankar, R. et al. Low-Threshold Lasing from 2D Homologous Organic-Inorganic Hybrid Ruddlesden-Popper Perovskite Single Crystals. Nano Lett. 2018, 18, 3221-3228.

(14) Milot, R. L.; Eperon, G. E.; Snaith, H. J.; Johnston, M. B.; Herz, L. M. Temperature-Dependent Charge-Carrier Dynamics in $\mathrm{CH}_{3} \mathrm{NH}_{3} \mathrm{PbI}_{3}$ Perovskite Thin Films. Adv. Funct. Mater. 2015, 25, 6218-6227.

(15) Wang, J.; Leng, J.; Liu, J.; He, S.; Wang, Y.; Wu, K.; Jin, S. Engineered Directional Charge Flow in Mixed Two-Dimensional Perovskites Enabled by Facile Cation-Exchange. J. Phys. Chem. C 2017, 121, 21281-21289.

(16) Liu, J.; Leng, J.; Wu, K.; Zhang, J.; Jin, S. Observation of Internal Photoinduced Electron and Hole Separation in Hybrid Two-Dimentional Perovskite Films. J. Am. Chem. Soc. 2017, 139, 1432-1435.

(17) Gélvez-Rueda, M. C.; Hutter, E. M.; Cao, D. H.; Renaud, N.; Stoumpos, C. C.;
Hupp, J. T.; Savenije, T. J.; Kanatzidis, M. G.; Grozema, F. C. Interconversion Between Free Charges and Bound Excitons in 2D Hybrid Lead Halide Perovskites. J. Phys. Chem. C 2017, 121, 26566-26574.

(18) Shang, Q.; Wang, Y.; Zhong, Y.; Mi, Y.; Qing, L.; Zhao, Y.; Qiu, X.; Liu, X.; Zhang, Q. Unveiling Structure Engineered Carrier Dynamics in Hybrid Quasi-TwoDimensional Perovskites Thin Film Towards Controllable Emission. J. Phys. Chem. Lett. 2017, 8, 4431-4438.

(19) Xing, G.; Wu, B.; Wu, X.; Li, M.; Du, B.; Wei, Q.; Guo, J.; Yeow, E. K. L.; Sum, T. C.; Huang, W. Transcending the slow bimolecular recombination in leadhalide perovskites for electroluminescence. Nat. Commun. 2017, 8, 14558.

(20) Venkatesan, N. R.; Labram, J. G.; Chabinyc, M. L. Charge-Carrier Dynamics and Crystalline Texture of Layered Ruddlesden-Popper Hybrid Lead Iodide Perovskite Thin Films. ACS Energy Lett. 2018, 380-386.

(21) Zhang, Y.; Wang, R.; Li, Y.; Wang, Z.; Hu, S.; Yan, X.; Zhai, Y.; Zhang, C.; Sheng, C. Optical Properties of Two-Dimensional Perovskite Films of $\left(\mathrm{C}_{6} \mathrm{H}_{5} \mathrm{C}_{2} \mathrm{H}_{4} \mathrm{NH}_{3}\right)_{2}\left[\mathrm{PbI}_{4}\right]$ and $\left(\mathrm{C}_{6} \mathrm{H}_{5} \mathrm{C}_{2} \mathrm{H}_{4} \mathrm{NH}_{3}\right)_{2} \quad\left(\mathrm{CH}_{3} \mathrm{NH}_{3}\right)_{2}\left[\mathrm{~Pb}_{3} \mathrm{I}_{10}\right] . J$. Phys. Chem. Lett. 2018, 13-19.

(22) Blancon, J.-C.; Tsai, H.; Nie, W.; Stoumpos, C. C.; Pedesseau, L.; Katan, C.; Kepenekian, M.; Soe, C. M. M.; Appavoo, K.; Sfeir, M. Y. et al. Extremely efficient internal exciton dissociation through edge states in layered 2D perovskites. Science 2017, 355, 1288-1292.

(23) Guo, Z.; Wu, X.; Zhu, T.; Zhu, X.; Huang, L. Electron-Phonon Scattering in Atomically Thin 2D Perovskites. ACS Nano 2016, 10, 9992-9998. 
(24) Chen, X.; Lu, H.; Li, Z.; Zhai, Y.; Ndione, P. F.; Berry, J. J.; Zhu, K.; Yang, Y.; Beard, M. C. Impact of Layer Thickness on the Charge Carrier and Spin Coherence Lifetime in Two-Dimensional Layered Perovskite Single Crystals. ACS Energy Lett. 2018, 3, 2273-2279.

(25) Wu, X.; Trinh, M. T.; Zhu, X.$Y$. Excitonic Many-Body Interactions in Two-Dimensional Lead Iodide Perovskite Quantum Wells. J. Phys. Chem. C 2015, 119, 14714-14721.

(26) Huang, L.; Krauss, T. D. Quantized Bimolecular Auger Recombination of Excitons in Single-Walled Carbon Nanotubes. Phys. Rev. Lett. 2006, 96.

(27) Wang, F.; Dukovic, G.; Knoesel, E.; Brus, L. E.; Heinz, T. F. Observation of rapid Auger recombination in optically excited semiconducting carbon nanotubes. Phys. Rev. B 2004, 70.

(28) Klimov, V. I. Quantization of Multiparticle Auger Rates in Semiconductor Quantum Dots. Science 2000, 287, 1011-1013.

(29) Bae, W. K.; Padilha, L. A.; Park, Y.-S.; McDaniel, H.; Robel, I.; Pietryga, J. M.; Klimov, V. I. Controlled Alloying of the Core-Shell Interface in CdSe/CdS Quantum Dots for Suppression of Auger Recombination. ACS Nano 2013, 7, 34113419 .

(30) Htoon, H.; Hollingsworth, J. A.; Dickerson, R.; Klimov, V. I. Effect of Zero- to One-Dimensional Transformation on Multiparticle Auger Recombination in Semiconductor Quantum Rods. Phys. Rev. Lett. 2003, 91 .

(31) Robel, I.; Bunker, B. A.; Kamat, P. V.; Kuno, M. Exciton Recombination Dynamics in CdSe Nanowires: Bimolecular to Three-Carrier Auger Kinetics. Nano Lett. 2006, 6, 1344-1349.

(32) Sun, D.; Rao, Y.; Reider, G. A.; Chen, G.; You, Y.; Brézin, L.; Harutyunyan, A. R.;
Heinz, T. F. Observation of Rapid Exciton-Exciton Annihilation in Monolayer Molybdenum Disulfide. Nano Lett. 2014, 14, 5625-5629.

(33) Kumar, N.; Cui, Q.; Ceballos, F.; He, D.; Wang, Y.; Zhao, H. Exciton-exciton annihilation in $\mathrm{MoSe}_{2}$ monolayers. Phys. Rev. B 2014, 89 .

(34) Yuan, L.; Huang, L. Exciton dynamics and annihilation in $\mathrm{WS}_{2} 2 \mathrm{D}$ semiconductors. Nanoscale 2015, 7, 7402-7408.

(35) Surrente, A.; Mitioglu, A. A.; Galkowski, K.; Klopotowski, L.; Tabis, W.; Vignolle, B.; Maude, D. K.; Plochocka, P. Onset of exciton-exciton annihilation in single-layer black phosphorus. Phys. Rev. B 2016, 94.

(36) Bae, W. K.; Park, Y.-S.; Lim, J.; Lee, D.; Padilha, L. A.; McDaniel, H.; Robel, I.; Lee, C.; Pietryga, J. M.; Klimov, V. I. Controlling the influence of Auger recombination on the performance of quantumdot light-emitting diodes. Nat. Commun. 2013, 4 .

(37) Kioupakis, E.; Rinke, P.; Delaney, K. T.; de Walle, C. G. V. Indirect Auger recombination as a cause of efficiency droop in nitride light-emitting diodes. Appl. Phys. Lett. 2011, 98, 161107.

(38) Guo, W.; Zhang, M.; Bhattacharya, P.; Heo, J. Auger Recombination in IIINitride Nanowires and Its Effect on Nanowire Light-Emitting Diode Characteristics. Nano Lett. 2011, 11, 1434-1438.

(39) Schaller, R. D.; Klimov, V. I. High Efficiency Carrier Multiplication in $\mathrm{PbSe}$ Nanocrystals: Implications for Solar Energy Conversion. Phys. Rev. Lett. 2004, 92.

(40) Li, M.; Begum, R.; Fu, J.; Xu, Q.; Koh, T. M.; Veldhuis, S. A.; Grätzel, M.; Mathews, N.; Mhaisalkar, S.; Sum, T. C. 
Low threshold and efficient multiple exciton generation in halide perovskite nanocrystals. Nat. Commun. 2018, 9 .

(41) Peng, W.; Yin, J.; Ho, K.-T.; Ouellette, O.; De Bastiani, M.; Murali, B.; El Tall, O.; Shen, C.; Miao, X.; Pan, J. et al. Ultralow Self-Doping in Two-dimensional Hybrid Perovskite Single Crystals. Nano Lett. 2017, 17, 4759-4767.

(42) Katan, C.; Mercier, N.; Even, J. Quantum and Dielectric Confinement Effects in Lower-Dimensional Hybrid Perovskite Semiconductors. Chem. Rev. 2019,

(43) Gauthron, K.; Lauret, J. S.; Doyennette, L.; Lanty, G.; Al Choueiry, A.; Zhang, S. J.; Brehier, A.; Largeau, L.; Mauguin, O.; Bloch, J. et al. Optical spectroscopy of two-dimensional layered $\left(\mathrm{C}_{6} \mathrm{H}_{5} \mathrm{C}_{2} \mathrm{H}_{4} \mathrm{NH}_{3}\right)_{2}-\mathrm{PbI}_{4}$ perovskite. Opt. Express 2010, 18, 5912-5919.

(44) Pelant, I.; Valenta, J. Luminescence Spectroscopy of Semiconductors; Oxford University Press, 2012.

(45) Ref. 44, pp. 88-89.

(46) Abdel-Baki, K.; Boitier, F.; Diab, H.; Lanty, G.; Jemli, K.; Lédée, F.; Garrot, D.; Deleporte, E.; Lauret, J. Exciton dynamics and non-linearities in twodimensional hybrid organic perovskites. $J$. Appl. Phys. 2016, 119, 064301.

(47) Saba, M.; Cadelano, M.; Marongiu, D.; Chen, F.; Sarritzu, V.; Sestu, N.; Figus, C.; Aresti, M.; Piras, R.; Lehmann, A. G. et al. Correlated electron-hole plasma in organometal perovskites. Nat. Commun. 2014, 5, 5049 .

(48) Yamada, Y.; Nakamura, T.; Endo, M.; Wakamiya, A.; Kanemitsu, Y. Photocarrier Recombination Dynamics in Perovskite $\mathrm{CH}_{3} \mathrm{NH}_{3} \mathrm{PbI}_{3}$ for Solar Cell Applications. J. Am. Chem. Soc. 2014, 136, $11610-11613$.
(49) Giorgi, G.; Yamashita, K.; Palummo, M. On the Nature of the Electronic and Optical Excitations of Ruddlesden-Popper Hybrid Organic-Inorganic Perovskites: the Role of Many-Body Interactions. J. Phys. Chem. Lett. 2018, 9, 5891-5896.

(50) Iwata, Y.; Banal, R. G.; Ichikawa, S.; Funato, M.; Kawakami, Y. Emission mechanisms in Al-rich $\mathrm{AlGaN} / \mathrm{AlN}$ quantum wells assessed by excitation power dependent photoluminescence spectroscopy. $J$. Appl. Phys. 2015, 117, 075701.

(51) Leng, K.; Abdelwahab, I.; Verzhbitskiy, I.; Telychko, M.; Chu, L.; Fu, W.; Chi, X.; Guo, N.; Chen, Z.; Chen, Z. et al. Molecularly thin two-dimensional hybrid perovskites with tunable optoelectronic properties due to reversible surface relaxation. Nat. Mater. 2018, 17, 908-914.

(52) Fang, H.-H.; Yang, J.; Tao, S.; Adjokatse, S.; Kamminga, M. E.; Ye, J.; Blake, G. R.; Even, J.; Loi, M. A. Unravelling Light-Induced Degradation of Layered Perovskite Crystals and Design of Efficient Encapsulation for Improved Photostability. Adv. Funct. Mater. 2018, 1800305.

(53) Wu, X.; Trinh, M. T.; Niesner, D.; Zhu, H.; Norman, Z.; Owen, J. S.; Yaffe, O.; Kudisch, B. J.; Zhu, X. Trap States in Lead Iodide Perovskites. J. Am. Chem. Soc. 2015, 137, 2089-2096.

(54) Zhang, Z.; Fang, W.; Tokina, M.; Long, R.; Prezhdo, O. V. Rapid Decoherence Suppresses Charge Recombination in Multi-Layer 2D Halide Perovskites: TimeDomain Ab Initio Analysis. Nano Lett. 2018, 18, 2459-2466.

(55) Klingshirn, C. F. Semiconductor Optics; Springer Berlin Heidelberg, 2012.

(56) Lanty, G.; Jemli, K.; Wei, Y.; Leymarie, J.; Even, J.; Lauret, J.-S.; Deleporte, E. Room-Temperature Optical Tunability and Inhomogeneous Broadening in 2D-Layered Organic-Inorganic 
Perovskite Pseudobinary Alloys. J. Phys. Chem. Lett. 2014, 5, 3958-3963.

(57) Ishihara, T.; Hong, X.; Ding, J.; Nurmikko, A. Dielectric confinement effect for exciton and biexciton states in PbI4-based two-dimensional semiconductor structures. Surf. Sci. 1992, 267, 323326 .

(58) Kato, Y.; Ichii, D.; Ohashi, K.; Kunugita, H.; Ema, K.; Tanaka, K.; Takahashi, T.; Kondo, T. Extremely large binding energy of biexcitons in an organic-inorganic quantum-well material $\left(\mathrm{C}_{4} \mathrm{H}_{9} \mathrm{NH}_{3}\right)_{2} \mathrm{PbBr}_{4}$. Solid State Commun. 2003, 128, 15-18.

(59) Thouin, F.; Neutzner, S.; Cortecchia, D.; Dragomir, V. A.; Soci, C.; Salim, T.; Lam, Y. M.; Leonelli, R.; Petrozza, A.; Kandada, A. R. S. et al. Stable biexcitons in two-dimensional metal-halide perovskites with strong dynamic lattice disorder. Phys. Rev. Materials 2018, 2.

(60) Kondo, T.; Azuma, T.; Yuasa, T.; Ito, R. Biexciton lasing in the layered perovskitetype material $\left(\mathrm{C}_{6} \mathrm{H}_{13} \mathrm{NH}_{3}\right)_{2} \mathrm{PbI}_{4}$. Solid State Commun. 1998, 105, 253-255.

(61) Zhang, X.; Wu, G.; Fu, W.; Qin, M.; Yang, W.; Yan, J.; Zhang, Z.; Lu, X.; Chen, H. Orientation Regulation of Phenylethylammonium Cation Based 2D Perovskite Solar Cell with Efficiency Higher Than 11\%. Adv. Energy Mater. 2018, 1702498.

(62) Feldmann, J.; Peter, G.; Göbel, E. O.; Dawson, P.; Moore, K.; Foxon, C.; Elliott, R. J. Linewidth dependence of radiative exciton lifetimes in quantum wells. Phys. Rev. Lett. 1987, 59, 2337-2340.

(63) Wang, G.; Chernikov, A.; Glazov, M. M.; Heinz, T. F.; Marie, X.; Amand, T.; Urbaszek, B. Colloquium : Excitons in atomically thin transition metal dichalcogenides. Rev. Mod. Phys. 2018, 90.
(64) Amani, M.; Lien, D.-H.; Kiriya, D.; Xiao, J.; Azcatl, A.; Noh, J.; Madhvapathy, S. R.; Addou, R.; KC, S.; Dubey, M. et al. Near-unity photoluminescence quantum yield in $\mathrm{MoS}_{2}$. Science 2015, 350, 1065-1068.

(65) Jin, C.; Kim, J.; Wu, K.; Chen, B.; Barnard, E. S.; Suh, J.; Shi, Z.; Drapcho, S. G.; Wu, J.; Schuck, P. J. et al. On Optical Dipole Moment and Radiative Recombination Lifetime of Excitons in $\mathrm{WSe}_{2}$. Adv. Funct. Mater. 2016, 27, 1601741.

(66) Yu, Y.; Yu, Y.; Xu, C.; Barrette, A.; Gundogdu, K.; Cao, L. Fundamental limits of exciton-exciton annihilation for light emission in transition metal dichalcogenide monolayers. Phys. Rev. B 2016, 93.

(67) Yuan, L.; Wang, T.; Zhu, T.; Zhou, M.; Huang, L. Exciton Dynamics, Transport, and Annihilation in Atomically Thin TwoDimensional Semiconductors. J. Phys. Chem. Lett. 2017, 8, 3371-3379.

(68) Liu, Y.; Xiao, H.; Goddard, W. A. TwoDimensional Halide Perovskites: Tuning Electronic Activities of Defects. Nano Lett. 2016, 16, 3335-3340.

(69) Pandey, M.; Jacobsen, K. W.; Thygesen, K. S. Band Gap Tuning and Defect Tolerance of Atomically Thin TwoDimensional Organic-Inorganic Halide Perovskites. J. Phys. Chem. Lett. 2016, 7, 4346-4352. 